\title{
Harmonic emission assessment on a distribution network: the opportunity for the prevailing angle in harmonic phasors
}

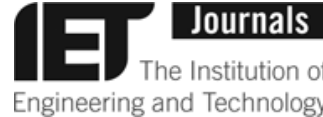

ISSN 2515-0855 doi: 10.1049/oap-cired.2017.0891 www.ietdl.org

\author{
Brandon Peterson ${ }^{1} \bowtie$, Johan Rens ${ }^{1}$, Jan Desmet ${ }^{2}$ \\ ${ }^{1}$ Faculty of Engineering: School of Electrical, Electronic and Computer Engineering, \\ North West University, Potchefstroom, South Africa \\ ${ }^{2}$ Faculty of Engineering and Architecture: Ghent University, Kortrijk, Belgium \\ E-mail: brandonbpeterson@gmail.com
}

\begin{abstract}
Harmonic emission assessment in high-voltage networks with a number of renewable power plants (RPP) interconnected is challenging. A sound scientific methodology readily accessible to engineers is needed to validate the compliance to grid code requirement set by the distribution system operator. Harmonic phasors recorded coherently all over the network can cause an impractical volume of data. This study investigates the opportunity to improve existing methodologies by application of the prevailing angle in a harmonic phasor as an approach to significant reduction of data and then demonstrate the evaluation of grid compliance in a network with a number of RPP interconnected. It is shown that the unrelated dynamic nature of the different non-linear energy sources does compromise the practical application of the prevailing harmonic phase angle. It is then concluded that the dynamic nature of RPP necessitates continuous monitoring of grid code requirements on harmonic emission.
\end{abstract}

\section{Introduction}

Distribution system operators (DSOs) are integrating large-scale renewable power plants (RPP) into their networks, as part of a drive to reduce their reliance on fossil fuels for the generation of electrical energy. As part of the DSO's grid code requirements, these RPPs have to prove compliance to a predetermined set of harmonic emission limits determined by the DSO. While the topic of harmonic emission's assessment has been widely discussed [1-3], the challenge remains to evaluate the compliance to harmonic emission limits pragmatically [4]. Some RPPs such as photovoltaic (PV) power plants are considered as a source of harmonics [5] due to the inverter technology employed in the electricity generation process.

CIGRE/CIRED C4.109 [6] proposed a method where a statistical approach is taken to the assessment of harmonic emissions using 10 min aggregated data in line with IEC 61000-4-30 [7]. Using single point measurements it has been shown that his method [8] does not yield conclusive results and can lead to incorrect assessment of harmonic emissions from an RPP.

Harmonic phasor data is the preferred method of harmonic emission assessment $[9,10]$; however, the volume of data resulting is restrictive and time consuming. Engineers require a pragmatic approach to the assessment of harmonic emission from RPPs.

Aggregation of harmonic phasors, albeit a misnomer in principle has been shown [11] to add value in the assessment of harmonic emission assessment. This paper applies the aggregation of harmonic phasor principles on a high-voltage distribution network were multiple PV plants, as well as traction loads are integrated into the same network. The aim is to highlight the dynamic nature of the non-linear loads connected to the same network and how this may be a challenge for RPPs attempting to prove grid code compliance.

\section{Harmonic phasors aggregation}

Harmonic phasors are used to simplify the analysis of voltage and current waveforms distortion in the frequency domain. IEC
61000-4-30 methods make use of a continuous measurement of a voltage or current waveform and then integrate the data into $200 \mathrm{~ms}$ data blocks. The $200 \mathrm{~ms}$ data blocks are then aggregated into $3 \mathrm{~s}$ data blocks, which are then integrated into $10 \mathrm{~min}$ data points as visualised in Fig. 1. Aggregation beyond the $200 \mathrm{~ms}$ data blocks only retains the RMS data and all other phasor data such as the phase angle information is lost.

In line with IEC 61000-4-30 principles, the aggregation of harmonic phasors to retain the phase angle information is proposed in $[13,14]$. The approach thus far has been limited to the application of measurements on low-voltage and medium-voltage systems [11], but has yielded value for in the assessment of harmonic emission.

The aggregated harmonic phasor $Y_{\mathrm{agg}, h}$ at harmonic $h$ is derived from the $200 \mathrm{~ms}$ data blocks over a specified time period

$$
Y_{\mathrm{agg}, h}=Y_{\mathrm{agg}, h} \angle \varphi_{\mathrm{agg}, h}
$$

where $Y_{\mathrm{agg}, h}$ is the RMS value of the aggregated harmonic voltage or current phasor at harmonic $h$ using an aggregation period of $1 \mathrm{~min}$ and $\varphi_{\text {agg, } h}$ is the aggregated harmonic voltage or current phase angle with $h$ the harmonic number. .

$Y_{\text {agg, } h}$ is calculated as follows:

$$
Y_{\mathrm{agg}, h}=\left(\frac{1}{N} \sum_{i=1}^{N} Y_{200 \mathrm{~ms}, h, i}^{2}\right)^{1 / 2}
$$

and $\varphi_{\text {agg }, h}$ is calculated as follows:

$$
\varphi_{\text {agg }, h}=\arg \left(\sum_{i=1}^{N} Y_{H, h, i}\right)
$$

Harmonic phasors can vary from one $200 \mathrm{~ms}$ sample to the next due to the dynamic nature of load or DSO network. To determine if the aggregated harmonic phasor data is usable the 'level of prevalence' is calculated to evaluate the variance between samples $[13,14]$ using 


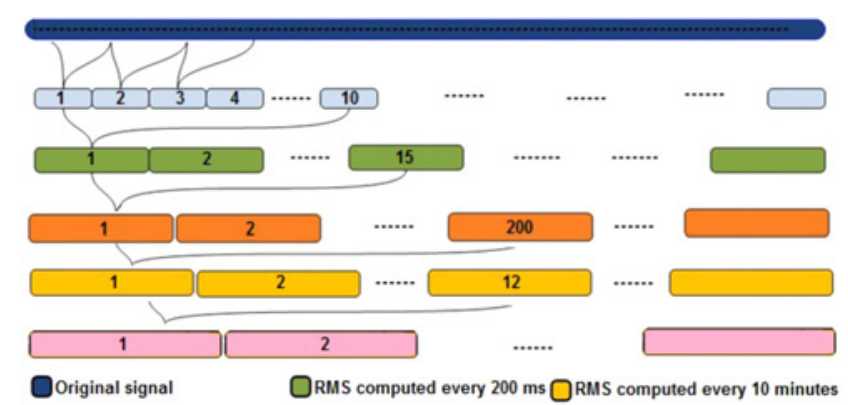

ORMS computed every period ORMS computed every $3 \mathrm{~s}$ ORMS computed every 2 hours

Fig. 1 IEC 61000-4-30 aggregation principles [12]

the ratio (4) of the harmonic phasor vs the RMS harmonic value. A prevalence level of $>0.95$ is considered to have a high similarity and the data may be used, as such a single value may be used to represent the harmonic phasor data collected. This single value is referred to as the prevailing harmonic phase phasor. A prevalence level of $<0.8$ has little prevalence and the data may not be used, thus no single value may be used to determine the harmonic phasor data

$$
\mathrm{PR}_{\mathrm{agg}, h}=\frac{\left|\sum_{i=1}^{n} Y_{H, h, i}\right|}{\sum_{i=1}^{n}\left|Y_{H, h, i}\right|}
$$

\section{Field application in DSO network}

Multiple power quality (PQ) recorders, with the ability to aggregate the harmonic phasor data, were installed in a distribution high-voltage network as shown in Fig. 2 to explore the concept of the prevailing phasor. The PQ recorders were all GPS time synchronised for coherent measurements with a time uncertainty better than $1 \mu \mathrm{s}$.

\subsection{Aggregated harmonic phasors}

The analysis of the results is limited to the fifth and seventh harmonic. As both the RMS value and the corresponding phase angle are retained in the aggregation process, the data is presented in polar plot format. The feeder number on the figures corresponds to the PQ recorder number as indicated in Fig. 2.

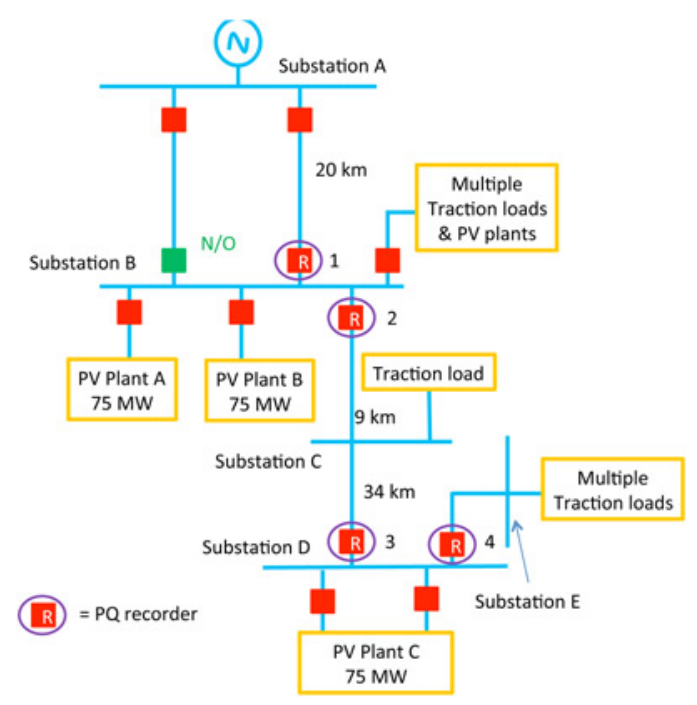

Fig. $2132 \mathrm{kV}$ DSO network with $P Q$ recorders

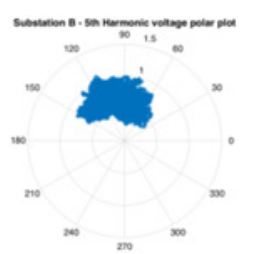

Fig. 3 Substation B fifth voltage harmonic

Figs. 3-6 represent the fifth and seventh voltage harmonic as measured on the $132 \mathrm{kV}$ busbars at substation B and substation D. It is observed that the harmonic phasors are located in multiple quadrants, highlighting the net effect of the distorting harmonic loads present in the network. Harmonic voltages are a result of the non-linear harmonic currents being drawn through the network impedance [15] and why the assessment of harmonic emission is mostly a 'current-based' approach.

Figs. 7-14 are polar plots for the fifth and seventh current harmonics as measured at the various nodes. Note that most of the harmonic phasors are located in more than two quadrants in some instances, highlighting their dynamic nature within this high-voltage network. It is has been shown in [14] that if one source of harmonics exist within a network the harmonic current phasors would be localised in one quadrant and in some cases partially sharing with another quadrant.

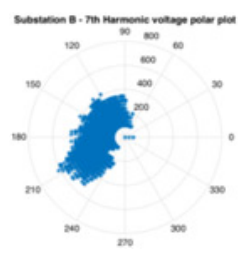

Fig. 4 Substation B seventh voltage harmonic

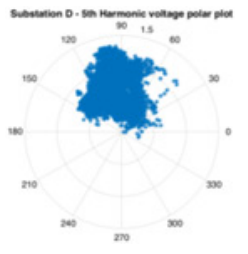

Fig. 5 Substation D fifth voltage harmonic

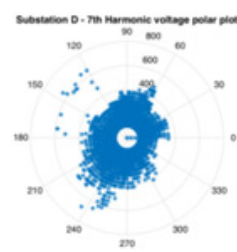

Fig. 6 Substation D seventh voltage harmonic

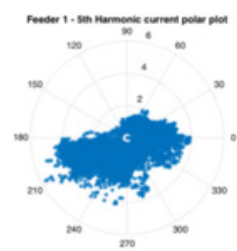

Fig. 7 Feeder 1 fifth current harmonic 


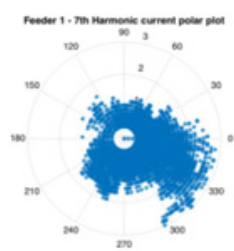

Fig. 8 Feeder 1 seventh current harmonic

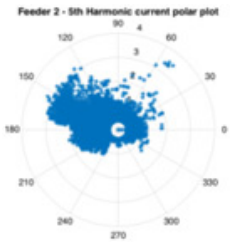

Fig. 9 Feeder 2 fifth current harmonic

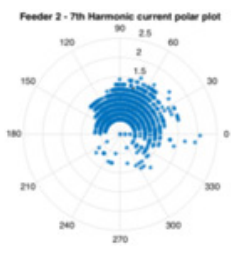

Fig. 10 Feeder 2 seventh current harmonic

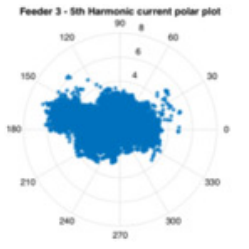

Fig. 11 Feeder 3 fifth current harmonic

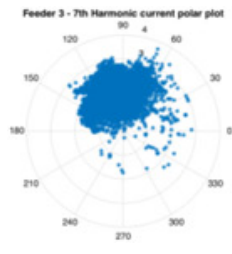

Fig. 12 Feeder 3 seventh current harmonic

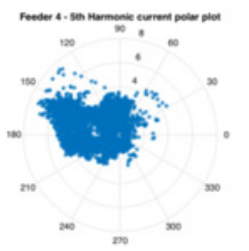

Fig. 13 Feeder 4 fifth current harmonic

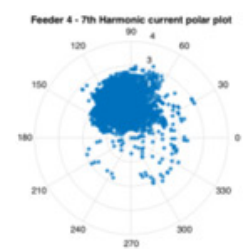

Fig. 14 Feeder 4 seventh current harmonic

\subsection{Level of prevalence}

The levels of prevalence for the voltage harmonics are listed in Table 1.

The levels of prevalence for the fifth harmonic voltage is above 0.9 indicating that the harmonic phasors are located in close proximity, although over two quadrants the values are mostly concentrated around the $90^{\circ}$ area.

Prevalence levels for the current harmonics are listed in Table 2.

Observe the reduction in the level of prevalence for the assessed current harmonics as the harmonic assessment moves up within the DSO network towards the source. This is testament to the number of distorting loads located in the DSO network, which all interact with each other within a very dynamic network. The erratic nature of the traction loads also add to the poor levels of prevalence, as the harmonic distortion will increase once a train goes past the respective supply point and reduces down to a floating load once the train has passed. The traction load and all the PV plants summate at substation B, which result in poor levels of prevalence.

Low levels of prevalence are an indication that the prevailing harmonic phase angle is not useful [13, 14]. The low levels of prevalence in this paper validate the dynamic nature of multiple distorting loads connected onto the same $132 \mathrm{kV}$ network. Due to the interaction of the different sources of harmonics being less when moving upstream towards higher fault levels, the level of prevalence in harmonic phase angle, improve.

\section{Harmonic emission assessment}

A challenge exists for RPPs to prove compliance to site-specific emission limits set by the DSO in line with IEC61000-3-6 [16]. A method (typically implemented within PQ recorders) for harmonic emission assessment is based on the direction of harmonic

Table 1 Level of prevalence - voltage harmonics

\begin{tabular}{lc}
\hline Substation & Prevalence factor fifth harmonic \\
\hline B & 0.913 \\
D & 0.933 \\
& \\
Substation & Prevalence factor seventh harmonic \\
B & 0.811 \\
D & 0.023 \\
\hline
\end{tabular}

Table 2 Level of prevalence-current harmonics

\begin{tabular}{lc}
\hline Feeder & Prevalence factor fifth harmonic \\
\hline 1 & 0.157 \\
2 & 0.663 \\
3 & 0.471 \\
& 0.772 \\
Feeder & \\
1 & Prevalence factor seventh harmonic \\
2 & 0.539 \\
4 & 0.889 \\
& 0.912 \\
\hline
\end{tabular}


active power [15] which is not useful when more than one distorting load exist in an interconnected network.

Pre- and post-connection assessment of harmonics as an alternative is detailed in IEC 61000-3-6. This is a relatively simple approach by measuring the harmonic phasors prior to the distorting load being connected and again after the load (source of distortion) is commissioned. The difference between the two sets of measurements is claimed to be the harmonic emission of the distorting load.

In the event that only RMS values are used, IEC 61000-3-6 suggests 'alpha' factors to summate the harmonic data within certain ranges. In this paper, it is clear that the background harmonic distortion influence the pre- and post-harmonic emission assessment due to the interaction of multiple distorting loads connected to the same $132 \mathrm{kV}$ network.

RPPs should only account for the harmonic currents they injected into the DSO network. Application of the IEC61000-3-6 approach resulted in the harmonic emission assessment not useful.

It has been shown [8] that harmonic measurements, if recorded synchronously at each node of interest, could improve the assessment of harmonic emission when based on the IEC 61000-3-6 approach. Some constraints remain as only RMS data is considered.

Coherent recordings at a number of points can improve grid code compliance monitoring as the harmonic prevalence phasor as demonstrated in this paper significantly reduce the volume of data to be analysed without compromising the integrity of the measured data when the level of prevalence is high.

\section{Conclusion}

With the aggregation of harmonic phasors, the volume of data required for harmonic emissions assessment is vastly reduced, which enables a practical engineer to conduct a harmonic emission assessment over longer periods without compromising data integrity.

In this paper, it has been shown that low levels of prevalence are an indication of the dynamic nature of multiple non-linear loads within the same high voltage DSO network. The low levels of prevalence nullify the prevailing phasor as a representative value for the harmonic distortion under evaluation.

RPPs requiring to prove compliance to the DSO's grid code conditions will encounter difficulties in proving compliance within a network such as in the case study. Suggested methods, as in IEC 61000-3-6, may prove to be inconclusive due to the dominance of the background harmonic distortion as such RPPs will have to employ alternative methods to ensure that the background harmonic distortion is excluded from their emissions assessment and to only account for the emissions which they emit onto the DSO network. Synchronised multiple point measurements is useful in the case where multiple distorting loads exist within the same network, in a discriminative approach to harmonic emission assessment of RPPs.

\section{References}

1 Bazina, M., Tomiša, T. 'Comparison of various methods for determining direction of harmonic distortion by measuring in point of common coupling'. ENERGYCON 2014-IEEE Int. Energy Conf., 2014, pp. 392-399

2 Blažič, B., Pfajfar, T., Papič, I.: 'A modified harmonic current vector method for harmonic contribution determination'. Power Systems Conf. and Exposition, 2004

3 Pfajfar, T., Papič, I.: 'Harmonic emission level estimation based on measurements at the point of evaluation'. IEEE Power and Energy Society General Meeting, 2011, pp. $1-5$

4 Peterson, B., Rens, J., Minnaar, U., et al.: 'A South African review of harmonic emission level assessment as per IEC61000-3-6'. CIGRE, 2015, pp. 1-9

5 Chicco, G., Schlabbach, J., Spertino, F.: 'Characterisation and assessment of the harmonic emission of grid-connected photovoltaic systems'. 2005 IEEE Russia Power Tech, PowerTech, 2005, pp. 1-7

6 Jaeger, E. D. E.: 'Disturbance emission level assessment techniques (CIGRE/ CIRED joint working group C4-109)'. 20th Int. Conf. Electricity Distribution, 2009, no. 470 , pp. $8-11$

7 IEC: 'IEC61000-4-30 testing and measurement techniques - power quality measurement methods' (Geneva, Switzerland, 2015)

8 Peterson, B., Rens, J., Botha, G., et al.: 'A discriminative approach to harmonic emission assessment'. IEEE Int. Workshop on Applied Measurements for Power Systems (AMPS), 2015, June

9 Bhattacharyya, S., Cobben, S., Ribeiro, P., et al.: 'Harmonic emission limits and responsibilities at a point of connection', IET Gener. Transm. Distrib., 2012, 6, (May 2011), p. 256

$10 \mathrm{Xu}, \mathrm{W}$. , Bahry, R., Mazin, H. E., et al.: 'A method to determine the harmonic contributions of multiple loads'. 2009 IEEE Power and Energy Society General Meeting (PES '09), 2009, pp. 1-6

11 Peterson, B., Rens, J., Meyer, J., et al. ' On the assessment of harmonic emission in distribution networks: opportunity for the prevailing harmonic phase angle'. IEEE Int. Workshop on Applied Measurements for Power Systems (AMPS), 2016, pp. 3-8

12 Calin, M., Dumitrescu, A. M., Asprou, M., et al.: 'Measurement data aggregation for active distribution networks', IEEE Int. Workshop on Applied Measurements for Power Systems (AMPS), 2013, pp. 144-149

13 Blanco, A. M., Stiegler, R., Meyer, J., et al.: 'Implementation of harmonic phase angle measurement for power quality instruments', IEEE Int. Workshop on Applied Measurements for Power Systems (AMPS), 2016, pp. 5-10

14 Meyer, J., Blanco Castaneda, A., Domagk, M., et al.: 'Assessment of prevailing harmonic current emission in public low voltage networks', IEEE Trans. Power Deliv., 2016, 8977, (c), pp. 1-1

15 Swart, P. H., van Wyk, J. D., Case, M. J.: 'On techniques for localization of sources producing distortion in three-phase networks', Eur. Trans. Electr. Power, 2007, 6, (6), pp. 391-396

16 IEC: 'IEC-61000-3-6 assessment of emission limits for the connection of distorting installations to MV, HV and EHV power systems' (Geneva, Switzerland, 2008) 